\title{
The Study on Recessive Financial Capital: "Value Twist Effect" Based Perspective
}

\author{
Deng Xiaojun \\ College of Economics \& Management \\ Xi'an Shiyou University \\ Xi'an Shaanxi 710065
}

\author{
Deng Jie \\ College of Economics \& Management \\ Beijing University of Chemical Technology \\ Beijing 100029
}

\begin{abstract}
This paper manages to explore the necessity and feasibility of the research of the recessive financial capital from the perspective of the "value twist effect", such as "distorting mirror effect", "funnel effect" and "WACC guillotine cutting effect" directed at the vacant research status of the recessive financial capital. For this purpose, based on the combing and analyzing the reference system of the current recessive financial capital research and the field research on the EVA enterprises performance evaluation's result of Petrochina Shaanxi Marketing Company and China Mobile Shaanxi Ltd, this paper has observed that the objective existing of "distorting mirror effect", "funnel effect" and "WACC guillotine cutting effect" in enterprise financial value decision practice is the root of enterprise value impairment and breakage. The enterprise value impairment and breakage caused by these "value twist effect" are usually unable to do the accurate valuation and measure or being reflected in the financial statement, which has reflected a kind of recessive value impairment and breakage. On one hand, the valuation of recessive value impairment and breakage has directly promoted the research of the recessive financial capital, on the other hand, the research of the recessive financial capital has provided the policy thoughts and methods for eliminating the "distorting mirror effect", "funnel effect" and "WACC guillotine cutting effect”.
\end{abstract}

Keywords-recessive financial capital; value twist effect, financial practice; driving force

\section{INTRODUCTION}

As the logic point of the research of finance which is analyzed through the three dimensions of principal, capital and enterprise value, the progress track of financial objective which is: output value maximum $\rightarrow$ profit maximization $\rightarrow$ enterprise value maximization; But the value and value creation in the view of finance and accounting indicates that: cost of capital and capital structure are the source of enterprise value and value creation; capital structure behavior is the origin of enterprise value endogenous creation, recessive financial capital is the kernel driving factor for the enterprise value creation in new economic era. Although the recessive capital and invisible benefit which are coexisted with dominant capital and dominant profit have uncertain financial value created by the recessive financial capital, the financial statements do not reflect, or hard to reflect, or cannot reflect them. However, it seems that it only affects the current financial value decision behavior and lowers the financial profit expectations of the rational stakeholders, and it does not bring to the enough attention on financial capital profit or loss for the rational stakeholders. This has directly caused the objective existence of "value twist effect" in the financial value decision practice. "Value twist effect" refers to the heteromorphism of value impairment and breakage of enterprise value created by recessive financial capital which may be caused by inadequate effectiveness or inherent limitation of current valuation measuring technologies in the financial value decision practice of the recessive financial capital. These are specifically manifested as the "distorting mirror effect", "funnel effect" and "WACC guillotine cutting effect".

\section{II. “Distorting MiRROR EFFECT” AND THE RESEARCH OF RECESSIVE FINANCIAL CAPITAL}

"Distorting mirror effect" resorts implicit employment effect of distorting the physical appearance through distorting mirror to depict or describe the heteromorphism of the distorting of enterprise economic benefit caused by the objective existence of recessive phenomenon and recessive cost. Gong Xikui (1992a, 1992b) [1] analyzed and combed the recessive phenomenon and recessive cost earlier, who classified the manifestation patterns of the recessive phenomenon in our actual economic life in our country into five categories: recessive unemployment, invisible income, implicit cost, implicit negotiation and recessive inflation. "Distorting mirror effect" is useful for analyzing the reasons of the occurrence of recessive phenomenon in our actual economic life in our country of different periods. The reasons lie in that the difference of economic goals and social goals set or expected by stakeholders of different levels in our country. Therefore, when the various interest demands which may be valid or invalid in a legal form of stakeholders of different levels cannot totally or partly realized, various economic conflicts and social conflicts will be occurred or concurred among them. In order to avoid various economic conflicts becoming social conflicts, coordinating, considering and realizing the part economic interests target of them will be the emergency policy measures to solve economic conflicts. Since the emergency policy measures is lacking of longevity and stability, its cost is the partial distorting of the laws of economics which are in healthy operation. And its result is that the partial covered and objective economic fact has caused the partial distorting of the economic information passed by economic target system, through which the recessive economic phenomena in real life have been formed. The existence of recessive economic phenomena does damage to the 
performance of our government's economic policies and the macro-control ability of the government economic levers and distorts the economic information provided by government economic reckoning target system in various degrees. All these have done direct damage to the economic reputation of our country, through which the reputation crises has formed; since the economic factors, such as unemployment, inflation and stakeholders' contract own the universal value of cross-cultural and cross-system, we should update the attitude and concept of unemployment and inflation which are of universal significance in governing the recessive economic phenomena in our country, as well as give full play to the coupled linkage of "invisible hand" of market mechanism and the "visible hand" of administrative macro-control policy.

\section{III. “FUNNEL EFFECT” AND THE RESEARCH OF RECESSIVE FINANCIAL CAPITAL}

"Funnel effect" is used to describe the recessive drain phenomena caused by the current system, mechanism of the man-made or unconscious behaviors in the value created by state-owned enterprises. [2]Zheng Chuanhai has described this phenomenon in his paper Implicit Cost: Funnel of Profit Drain at the earliest, who held that the existence of implicit cost has the implicit erosion for construction enterprises, which can be visually described by "funnel effect". The reasons of causing the existence of implicit cost in construction enterprises can be concluded as the following: the mobility of the field work in this special industry of construction enterprises forms the recessiveness of the variable cost accounting and measurement; there is hidden characteristics in the inaccurate measurement the influence towards regional environment and residents' living; diversification of the guide of market economy and social conflicts diversification caused by the field work in construction enterprises own the hidden characteristics. Ever since, researchers have made diversity analysis research on "funnel effect" of the recessive drain of enterprise value from different perspectives. For example, combined with the cost control practice of airline companies and compared with the explicit cost, Zhang Zheng (2004) [3]holds that the implicit cost is "usually difficult to find" in cost control practice and the off-sheet cost which has not disclosed in financial statements. And he classified the implicit cost of airline company into four categories: recessive strategic cost, recessive administration cost, recessive practice cost and recessive culture cost; Liu Yuan (2011) [4] holds that the implicit cost is hidden in the enterprise' $s$ total cost and free from the financial auditing supervision, which bears the characteristics of elusive, amplifying and explosive; [5]Xu Xiang (2011) classified the implicit cost into four categories: recessive mechanism cost, recessive institutional cost, recessive opportunity cost and recessive frictional cost. The different researches of recessive financial capital done by the above-mentioned scholars from different perspectives indicate that the capital gains and cost of the recessive financial capital is as same as that of the dominant financial capital, which have existed everywhere in the financial activities and financial relations of the creation of enterprise value in any moment. "Distorting mirror effect" of the distorting of economic benefits of enterprise and the "funnel effect" of recessive drain have highlighted the urgency of the research of recessive financial capital proposition.

\section{IV. “WACC GUILLOTINE CUTTING EFFECT” AND THE RESEARCH OF RECESSIVE FINANCIAL CAPITAL}

"WACC guillotine cutting effect" refers to the current system of examining and assessment and methods implement the "three don't consider principles" in the performance appraisal towards central enterprises owners in our country. The "three don't consider principles" are do not consider the nature and scale of the central enterprises, do not consider the difference between different industry of central enterprises and the regional social and economic development degree of central enterprises, do not consider the cost rate of debt capital and the cost rate of equity capital. Instead, the WACC is united into the cost rate of average capital, and it is classified into three grades: basis capital cost rate (5.5\%), lower capital cost rate $(4.1 \%)$ and floating capital cost rate (6.0\%). However, [6]Li Yangyang takes all the Shanghai and Shenzhen A-share listed companies of 13 industries in 2000-2009 as research sample and the statistical result has indicated that the average equity capital cost rate of the 13 industries in ten years in $9.61 \%$. Further, as for the cost rate of the average equity capital of the 13 industries in ten years, the average cost rate of agriculture, forestry, husbandry and fishery is 8.54; extractive industry is $13.33 \%$; manufacturing is $10.1 \%$; electricity, gas and water production and supply is 8.85\%; construction industry is $9.28 \%$; transportation and storage industry is $9.6 \%$; information technology industry is $8.52 \%$; wholesale and retail trade is $9.02 \%$; finance and insurance is $12.36 \%$; real estate is $10.06 \%$; social service industry is $7.81 \%$, communications and culture industry is $7.88 \%$ and comprehensive industry is $9.01 \%$. Compared with the basis capital cost rate $(5.5 \%)$ regulated by Operating Performance Evaluation Temporal Method of Responsible Person of Central Enterprise, the average equity capital cost rate decreases by $4.11 \%$; lower capital cost rate decreases by $5.51 \%$; floating capital cost rate decreases by $6.0 \%$ and the average capital cost decreases $4.41 \%$. The interval of the three low value mentioned-above is $2.61 \%$ $8.13 \%$, and the average of the low value is $5.37 \%$. This is the basic conclusion drew under the condition without considering the debt capital cost rate. If compared with WACC which has combines the debt capital cost rate, the low value of three capital cost rate regulated by Operating Performance Evaluation Temporal Method of Responsible Person of Central Enterprise will be larger, and the nest interval within the corresponding low value interval is $2.61 \%-8.13 \%$. Its corresponding average low value will be much higher than $5.37 \%$ undoubtedly.

Operating Performance Evaluation Temporal Method of Responsible Person of Central Enterprise in our country starts from October, 2003. By 2015, the State Owned Assets Supervision and Administration Commission of State Council has made three big revisions and implemented four evaluation of responsible person of central enterprises. In the four evaluations, the widely discredited and beleaguered element which ranks the first is always based on the three capital cost rate with the stiff form of design, lacking of elasticity and flexibility of the "three don't consider principles". That is the average equity capital cost rate, lower capital cost rate and floating capital cost rate. Do not consider the debt capital and the "guillotine cutting effect" of equity capital cost rate leads to 
a lower of the average capital cost rate in the performance evaluation. (The above-mentioned data indicate that the average low value is $5.37 \%$ ). The low average capital cost rate causes the false increase of EVA, thus the value creation ability of the responsible person of central enterprises and the value creation showed by EVA form are overestimated substantially. This has restrained the funding from debt financial partiality of the responsible person of central enterprises. And the design aim of the Operating Performance Evaluation Temporal Method of Responsible Person of Central Enterprise with the purpose of create incremental value maximization for owners becomes formalistic, and the ought-to-be function of the seriously distorted EVA evaluation system runs in the opposite direction with the essence of EVA.

The above analysis have indicates that the "WACC guillotine cutting effect" has also revealed the basic value orientation towards state-owned capital in the operating management activities of central enterprises: seldom considering or even do not consider the expected returns of the state-owned capital investment, misuse or exploit the investment capital, blindly pursuing the large-scale investment with diminishing marginal returns instead of considering the expected returns of capital project. The basic value orientation of the responsible person of central enterprises towards state owned capital, the heteromorphism of "funnel effect" profit drain existed in central enterprises (which emphasizes the explicit cost management and ignores the profit drain caused by the excavation and control of the implicit cost) and the heteromorphism of the "distorting mirror effect" existed in central enterprises (refers to the accounting information distortion revealed by the financial statements of central enterprises) have all highlighted the necessity and urgency of the research of recessive financial capital. The basic relationship is showing in the following fig. 1
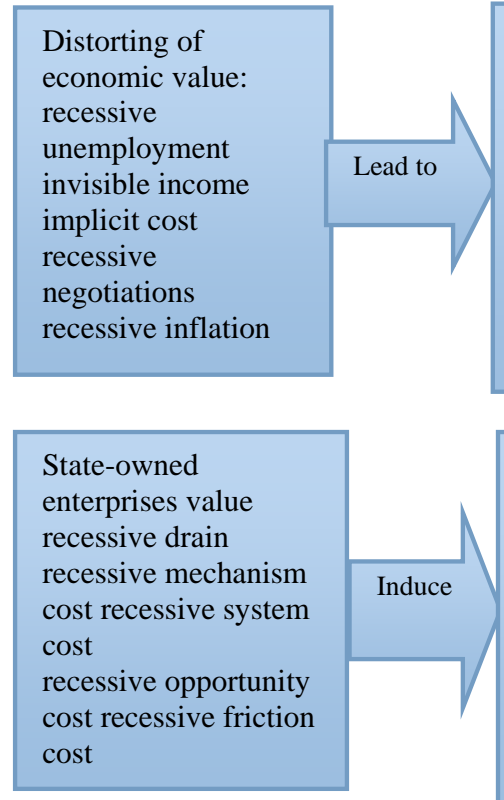

Performance evaluation of the responsible person in central enterprises: three don't consider principle average WACC undervaluation central enterprise EVA false increase the original intention of the design of EVA

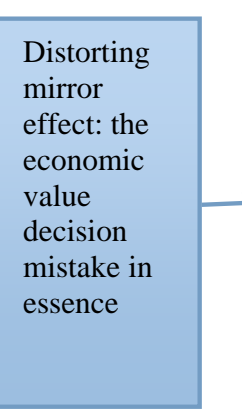

\section{Funnel} effect: the economic value decision mistake in essence
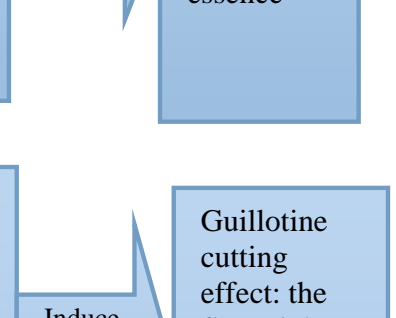

Guillotine cutting effect: the financial value decision practice distortion of enterprises in essence

Fig. 1. Figure "distorting mirror effect”, "funnel effect” and "guillotine cutting effect” with the research of recessive financial capital

\section{CONCLUSION}

1. The financial essence origins from the strategic decision of value creation maximization of stakeholders. Stakeholders 
pursue the maximization of self-investment capital value and the tactical game behavior which is chosen flexibly runs through every links of financial decision. No matter based on the logic analysis of financial theory, or the persistent financial value decision practice investigation, the different links or rational financial value decision have realized the financial essence target in the coupling optimization of explicit financial capital value creation and the appreciation of recessive financial capital value.

2. The reciprocal interdependence of explicit financial capital value and recessive financial capital value and the unbreakable characteristic of financial value creation have indicated that the emphatic trade studies in theory research must lead to the heteromorphism in financial value creation. In contrast, the mainstream financial researchers at home and abroad emphasize the research of explicit financial capital research and make light of the recessive financial capital research, which will lead to the existence of financial puzzles naturally, such as the capital structure puzzle, IPO puzzle, dividend and distribution puzzle. And it will also induce the "distorting mirror effect" which has distorted the financial value creation and the "funnel effect" of eroding the financial value-added, as well as the doubts of scientific, fair and objective fairness of EVA performance evaluation policy for the responsible person of central enterprise in our country in the financial practice.
3. Unveiling the financial theory heteromorphism abovementioned (capital structure puzzle, IPO puzzle, dividend and distribution puzzle) and eliminating the financial heteromorphism in the financial value decision practice ("distorting mirror effect" and "funnel effect") have highlighted the value and feasibility of recessive financial capital research.

\section{REFERENCES}

[1] Gong Xikui. Discussion Of The Recessive Phenomenon In Economy In Our Country [J]. Study \& Exploration, 1992 (3): 56-62. (In Chinese)

[2] Zheng Chuanhai. Implicit Cost: The Funnel Of Profit Drain [J]. Manager, 2006 (2): 48-49. (In Chinese)

[3] Zhang Zheng. Control The Recessive Cost, Improve Enterprise Profits [J]. Journal of Zhengzhou Institute of Aeronautical Industry Management, 2004 (12): 156-157. (In Chinese)

[4] Liu Yuan. The Analysis And Control Of Enterprise Recessive Cost [J]. Western Finance and Accounting, 2011 (8): 28-30. (In Chinese)

[5] Xu Xiang. Brief Analysis Of The Enterprise Recessive Cost Control [J]. Business Administrator, 2011 (4): 120. (In Chinese)

[6] Li Yangyang. The Technology Research Of The Cost Estimating Of The Prospective Equity Capital [D]. Beijing: Capital University of Economics and Business, 2013, p.55. (In Chinese)

[7] Harris, M.\&Raviv, A. Capital Structure and the Informational Role of Debt [J]. Journal of Finance, 1990 (45): 321-349. 